\title{
Corner kick effectiveness in the Greek Super League 2018-2019
}

\section{Mitrotasios ${ }^{a, *}$}

a School of Physical Education \& Sport Science, National \& Kapodistrian University of Athens, Athens, 172 37, Greece

*Corresponding author Ph: 2107276168 ; Fax: 2107276168 ; Email: micmit@phed.uoa.qr

DOI: https://doi.org/10.34256/ijpefs2134

Received: 15-06-2021, Revised: 08-07-2021; Accepted: 11-07-2021; Published: 12-07-2021

Abstract: The aim of the present study was to describe how corner kicks were taken and to identify key variables associated with final attempts during Greek Super League season 2018-19. There was a total of 424 final attempts after corner kick, of which, $16.3 \%$ were attempts off target, $6.8 \%$ attempts on target and $2.8 \%$ resulting in a goal. Bivariate analysis presented that attempts on target were increased when the ball was delivered outswing, no players positioned at the goal posts, $>1$ intervening attackers, attacking player the $1^{\text {st }}$ contact and ball delivered into the central zones of the 18-yard box. Binary logistic regression (Final attempt/ No Final attempt) revealed that the model was statistically significant for the final attempt $(p<0.001)$ and showed that the intervening attackers $(p<0.001)$ and the player of the 1st contact $(p<0.001)$ contributed significantly to the model. These results can aid coaches to enhance on one hand the attacking effectiveness; on the other hand the defensive efficacy of corner kicks within Greek football.

Keywords: Set plays, Soccer, Final attempt, Greek Super League

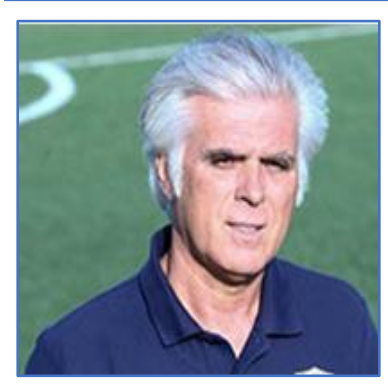

Dr. Mitrotasios has received a BSc degree from the Department of Physical Education and Sports Science of the Aristotle University of Thessaloniki. He has also awarded an MSc degree in training and coaching football and a PhD degree from German Sports University of Cologne. Currently he is working as a lecturer of Training and Coaching Football at the Department of Physical Education and Sports Science of the National and Kapodistrian University of Athens. His main research interests focus on athletic performance, GPS data analysis, match analysis and coaching.

\section{Introduction}

During a competitive football match, final attempts are originated from the two main situations of plays in the offensive phase. In open play, attempts are a consequent of ball possession from one of the teams; while in set play, final attempts result from restart situations, such as penalty kicks, free kicks, goal kicks and corner kicks [1]. Several studies that have analyzed set plays in high level competitive matches, presented that they contribute either directly or indirectly to about a third (30-37\%), of total goals scored [2-4], as well as they had a significant effect on the final result of the match [3]. Corner kicks account for $40-50 \%$ of the total set plays [5], while in a recent study examining World Cup 2018, found that $35.2 \%$ of goals scored derived from corner kicks [6]. This finding confirms an older study from Lago and his colleagues [7], who stated that the greatest portion of scored goals emanates from corner kicks in comparison to the rest of set plays types.

According to the Laws of the Game, corner kick is awarded to the attacking team, when a defender touches the ball last and as a result the ball goes out of goal line, beside or above the goalposts [8]. Tactically, a corner kick can be executed directly, when the ball is delivered direct into the 18-yard box or indirectly with at least one short pass, before the ball enters the 18-yard box [9]. In average 10 corners per match are performed. Concerning effectiveness, $2.2 \%$ of the corner kicks ended to goal scoring and this goal was responsible for the team winning or drawing the match on $76 \%$ of occasions, mainly at the first and the last 30 minutes of the match $[3,10]$. 
There is a significant number of studies which have analyzed the characteristics and the outcome of corner kicks. Casal and his colleagues [3] analysis of corner kicks from World Cup 2010, Euro 2012 and Champions League 2010-11 presented that more efficient corner kicks were executed directly (with cross) towards primarily the closest goalpost area. The ball was contended by one or two attackers and the defending team implemented organized defense with mixed marking.

Furthermore, de Baranda and his colleagues [10] examined 653 corner kicks from World Cup 2006 and presented that most corner kicks were executed by drawing teams. Moreover, the authors investigated the effect of match status on "type of kick", "zone of goal scored" and "defense near the goal". In regard with "type of kick" they found that corner kicks were performed with the opposite foot (outstep) [11], on inswing ball direction. According to Taylor and his colleagues [12] inswing corner kicks have greater chance to result in goal $(66 \%)$, in comparison to outswing kicks. Finally, tactical behavior of the defending team should be considered a critical factor for scoring, as in $80 \%$ [13] or $96 \%$ [10] of the cases mixed defense was deployed. Besides that, the analysis of goalpost marking presented that the majority of corner kicks included one defender positioned at the near post, fewer corner kicks included one player in each post and one defender at the far post. On the contrary, previous studies presented an increasing tendency, in which no defender is positioned near the two posts areas.

Concerning the number of players of attacking and defensive team during the corner kick, current literature shows the presence of at least six to seven defenders in the box, in comparison with attackers (four to five players). However, of greater significance is the number of attacking players involved for winning the ball, which is between one or two [3]. Moreover, efficiency of corner kicks has been correlated with the area in which the ball was crossed, as Pulling [9] and Grant and Williams [14] stated that corner kicks which were directed between the goal area and the penalty spot, led to more goals and final attempts target. Poon and his colleagues [15] alleged that the area with the greatest frequency of delivered corners ( $48 \%$ of total corners) was positioned centrally. Considering the aforementioned, the purpose of the present study was to investigate the characteristics of direct corner kicks and to define the variables related to corner's efficiency in Greek Super League 2018-19.

\section{Materials and Methods}

\subsection{Match sample}

The Greek Super League 2018-19 football season consisted of 240 matches, whereby 16 teams play against each other; once at a team's home stadium and once away at the opponent's stadium. A total, 1910 corner kicks were recorded, with 1600 of these being sampled as they satisfied the criterion of having the ball delivered directly into the box by the corner kick taker, while 310 that were delivered indirectly, were excluded from the study. Corner kicks were considered complete when the ball exited the 18yard box and did not immediately re-enter [16]. According to the Belmont Report [17] the use of public images for research purposes does not require informed consent or the approval of an ethical committee.

\subsection{Procedures}

Footage of the corners taken in sampled matches was downloaded from the Wyscout platform (Wyscout, Hudl, USA). Corner kicks were analysed using a custom notational instrument using Lince software [18]. The notational instrument was developed using the operational definitions selected from empirical research on corner kicks in the men's games $[3,9,16,19]$ and are outlined below:

1. Final result: a) Win: Sampled team scored more goals than opponent and won the match, b) Draw: Sampled team has scored equal goals to opponent and draw the match, c) Loss. Sampled team has scored fewer goals than opponent and lost the match.

2. Match status: a) Win: Sampled team scored more goals than opponent at the time of the corner kick, b) Draw. Sampled team has scored equal goals to opponent at the time of the corner kick, c) Loss. Sampled team has scored fewer goals than opponent at the time of the corner kick.

3. Half time: a) $1^{\text {st }}$ half. The corner kick was taken within 1st half time, b) $2^{\text {nd }}$ half: The corner kick was taken within $2^{\text {nd }}$ half time.

4. Ball direction: a) Inswing: The ball is sent towards the goal post, b) Outswing: The ball is sent outwards of the goal post. 
5. Defending goal post: a) Near post: A defending player stands at the near post, $b$ ) None: No defending player stands at the posts.

6. Type of marking: a) Zonal: All defending players in the effective play space (without considering the players of the post) were positioned at a particular spatial sector prior to the corner kick being taken. Defenders cover a particular space, b) Combined: Some defending players were positioned at a particular spatial sector and some were positioned against a specific member of the opposition team. Combination of zonal and man-to-man marking.

7. Intervening attackers: a) $0-1$ : None or one attacking player interacted with the ball, $b$ ) $>1$ : more than one attacking player interacted with the ball.

8. Defenders inside box: a) 1-8: up to 8 defensive players inside box, $b)>8$ : more than 8 defensive players inside box.

9. Attackers inside box: a) 1-5: Up to 5 attacking players inside box, b) $>5$ : more than 5 attacking players inside box.

10. Player of $1^{\text {st }}$ contact: a) Defender: a defender is the first player that contacted the ball, b) Attacker: an attacker is the first player that contacted the ball.

11. Final attempt: a) Yes. corner kick with final attempt, b) No: corner kick without final attempt.

12. Final attempt zones: The zone from which the final attempt was executed. Figure 1 depicts the 7 zones: Center $(\mathrm{Cn})$, Front (Ft), Near side (Ns), Far side (Fs), 1st post (P1), 2nd post (P2) and Out of box (Ob).

Outcome: a) Goal: The ball went over the goal line and into the net, b) Attempt on target: The ball would have entered the net, but prevented by goalkeeper or defender, c) Attempt off target. Attempt by the attacking team that was not directed within the dimensions of the goal. An attempt that made contact with the crossbar or either of the posts was classified as an attempt off target.

\subsection{Reliability}

To ensure the intra- and inter-reliability of the observational instrument, randomly $20 \%$ of the data were re-analyzed after 2 weeks (to exclude any learning effects) by the same observer (Ob1) and by different observer (Ob2).

Table 1. Intra- and inter-observer reliability.

\begin{tabular}{lll}
\hline Variable & $\begin{array}{l}\text { Intra-observer } \\
\text { Ob1 test - retest }\end{array}$ & $\begin{array}{l}\text { Inter-observer } \\
\text { Ob1 - Ob2 }\end{array}$ \\
\hline Final result & 1.00 & 1.00 \\
Match status & 1.00 & 1.00 \\
Half time & 1.00 & 1.00 \\
Ball direction & 0.97 & 0.95 \\
Defending goal post & 0.95 & 0.93 \\
Type of marking & 0.90 & 0.89 \\
Intervening attackers & 0.88 & 0.82 \\
Defenders inside box & 0.85 & 0.80 \\
Attackers inside box & 0.87 & 0.80 \\
Player of $1^{\text {st }}$ contact & 0.88 & 0.82 \\
Final attempt & 1.00 & 1.00 \\
Final attempt zone & 0.92 & 0.86 \\
Outcome & 0.96 & 0.91 \\
\hline Ktotal & 0.94 & 0.91
\end{tabular}




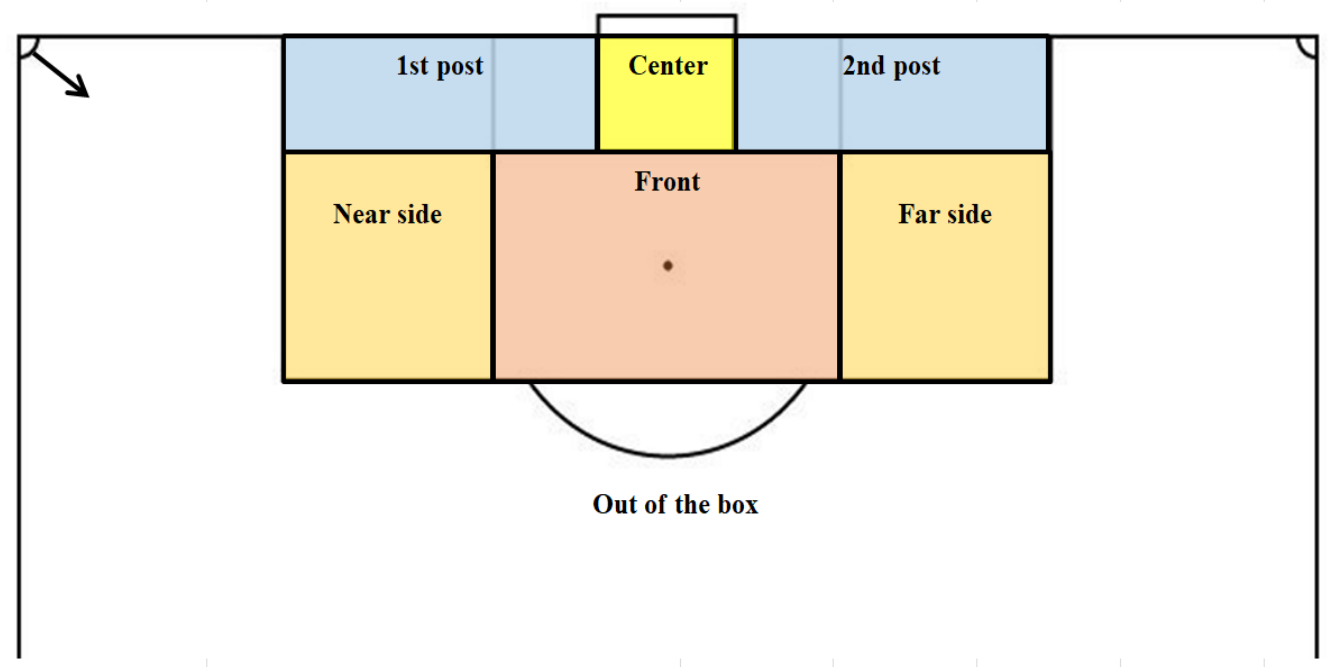

Figure 1. Final attempt zones.

The observers were trained in the use of the observational instrument following the protocols described by Losada \& Manolov [20]. They were experienced football coaches with more than $10 y e a r$ experience (Graduated in Sports Sciences, certified with UEFA Pro license). Kappa's coefficient was calculated for each variable and is presented in Table 1 , with mean kappa statistic of $k=0.93$ and $k=0.91$, classified as "perfect" intra- and inter-observer agreement, respectively [21].

\subsection{Statistical analysis}

For the statistical analysis SPSS v26 (IBM, USA) was used. Initially, descriptive statistics (means, frequencies, percentage) were used in to describe the characteristics of corner kicks (e.g., total number of kicks, corners per match and tactics used). A chisquare test of independence $\left(X^{2}\right)$ was used to examine the possible differences between the corner kicks with final attempt (FAtt) and without final attempt (NFAtt). In order to examine the size of the association between corner kicks' tactics variables and final attempt, the effect size $\varphi$ coefficient for the dichotomous variables and contingency coefficient (CC) for variables with more than two levels were used [22]. Finally, a binary logistic regression (Enter method) was conducted in order to examine which of the corner kicks' tactics variables are significant predictors for the final attempt in Greek Super League 2018-19. Similar process has also been followed by Casal and his colleagues [3] examining corner kicks in elite football tournaments. The significance level was set at $p<$ 0.05 .

\section{Results}

\subsection{Descriptive analysis}

A total of 1600 corner kicks were collected, an average of 6.7 per match. There was a total of 424 (26.5\%) final attempts after corner kick, of which, 267 $(16.7 \%)$ were attempts off target, 112 (7.0\%) attempts on target and $45(2.8 \%)$ resulting in a goal. At the Greek Super League 2018-19 most corner kicks were executed by winning teams $(42.2 \%)$, at second half $(55.4 \%)$ on a drawing match status $(52.4 \%)$. The most frequent ball direction was outswing kick (51.6\%). Teams on defense, most commonly used a player at near goalpost (55.3\%), combined marking $(93.6 \%)$ and more than eight players inside the box $(67.8 \%)$. On the other hand, attacking teams most commonly used more than one intervening attackers $(61.8 \%)$ and $1-5$ players inside the box $(67.6 \%)$. The most frequent player of $1^{\text {st }}$ contact with the ball was defending player $(67.5 \%)$ with the least frequently player being an attacker (32.5\%).

As far as spatial analysis is concerned, the zones of final attempt revealed significant differences between them $\left(x^{2}=515.83, p=0.000\right)$ as presented at Figure 2. In general, central zones were the most frequent used from teams in Greek League in order to execute FAtt, such as Center zone (11.8\%) and Front zone $(49.1 \%)$. Surprisingly, there was a major percentage of FAtt's that have been executed from outside the box (17.5\%).

\subsection{Bivariate analysis}

Results revealed significant differences between the corner kicks with final attmept and 
without final attempt (Table 2). In detail, there was a significant association for the likelihood of a FAtt for the winning teams (47.2\%, $\left.X^{2}=10.70, p=0.005\right)$. However, corner kick temporal analysis and match status were not associated with the creation of final attempts $\left(x^{2}=0.82, p=0.365\right.$ and $x^{2}=3.77, p=$ 0.155 respectively).

Moreover, there was a significant association for the likelihood of a FAtt when corner kick was executed with outswing direction $\left(59.7 \%, x^{2}=15.18\right.$, $p=0.000$ ). From a defensive point of view, there was a significant association for the likelihood of a NFAtt when teams had a player at near post $\left(57.6 \%, X^{2}=\right.$ $9.64, p=0.002)$ and player of $1^{\text {st }}$ contact was a defender $\left(83.2 \%, X^{2}=496.29, p=0.000\right)$. Concerning intervening attackers, there was a significant association for the likelihood of a FAtt when more than one attacker was involved in the set play $\left(343.30 \%, x^{2}\right.$ $=0.000, p=0.463$ ).

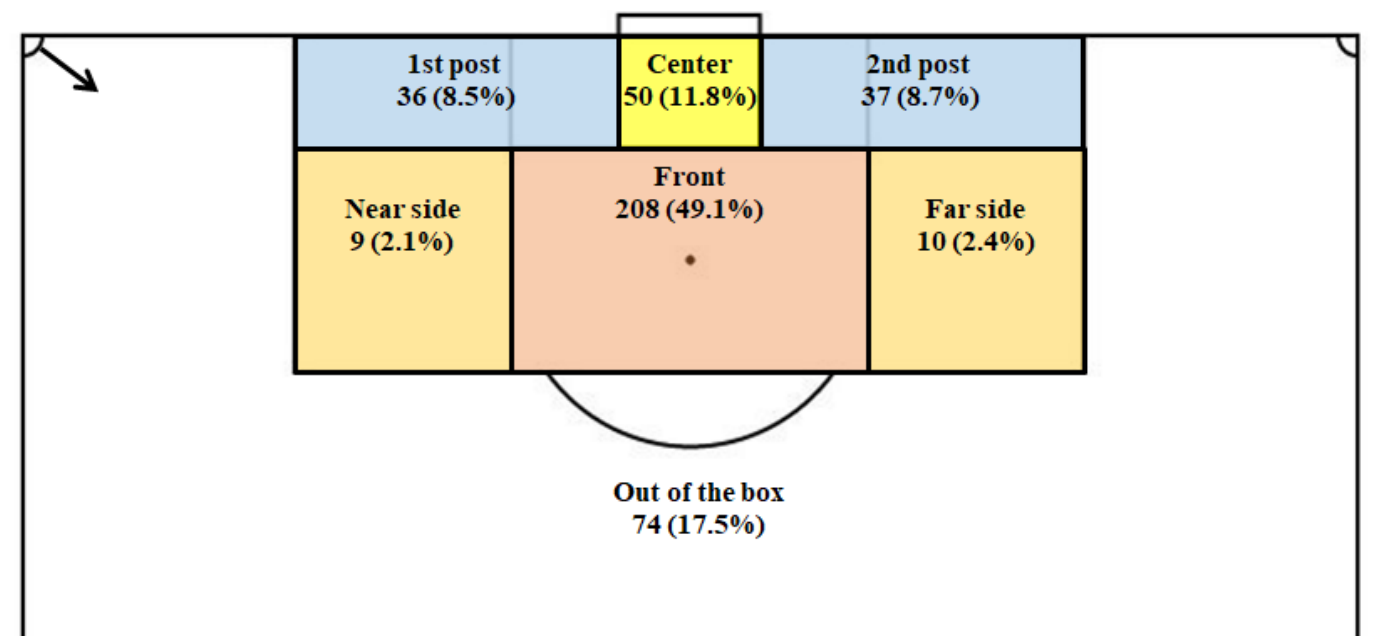

Figure 2. Final attempt zones from corner kicks in Greek SuperLeague 2018-19.

Table 2. Corner kicks effectiveness analyzed by attempt on target in Greek SuperLeague 2018-19.

\begin{tabular}{|c|c|c|c|c|c|c|c|c|c|c|}
\hline \multirow{2}{*}{ Variable } & \multirow{2}{*}{ Level } & \multicolumn{2}{|c|}{ NFAtt } & \multicolumn{2}{|c|}{ FAtt } & \multicolumn{2}{|l|}{ Total } & \multirow{2}{*}{$x^{2}$} & \multirow{2}{*}{ Sig } & \multirow[t]{2}{*}{$\begin{array}{l}\text { Effect size } \\
(\varphi \text { or } c c)\end{array}$} \\
\hline & & $\mathbf{n}$ & $\%$ & $\mathbf{n}$ & $\%$ & $\mathbf{n}$ & $\%$ & & & \\
\hline \multirow{3}{*}{ Final result } & Win & 475 & 40.4 & 200 & 47.2 & 675 & 42.2 & 10.70 & 0.005 & $C C=0.082$ \\
\hline & Draw & 284 & 24.1 & 110 & 25.9 & 394 & 24.6 & & & \\
\hline & Loss & 417 & 35.5 & 114 & 26.9 & 531 & 33.2 & & & \\
\hline \multirow{2}{*}{ Half time } & $1^{\text {st }}$ Half & 532 & 45.2 & 181 & 42.7 & 713 & 44.6 & 0.82 & 0.365 & $\varphi=-0.023$ \\
\hline & $2^{\text {nd }}$ Half & 644 & 54.8 & 243 & 57.3 & 887 & 55.4 & & & \\
\hline \multirow{3}{*}{ Match Status } & Win & 252 & 21.4 & 103 & 24.3 & 355 & 22.2 & 3.77 & 0.155 & $C C=0.048$ \\
\hline & Draw & 611 & 52.0 & 227 & 53.5 & 838 & 52.4 & & & \\
\hline & Loss & 313 & 26.6 & 94 & 22.2 & 407 & 25.4 & & & \\
\hline \multirow{2}{*}{ Ball direction } & Inswing & 604 & 51.4 & 171 & 40.3 & 775 & 48.4 & 15.18 & 0.000 & $\varphi=-0.097$ \\
\hline & Outswing & 572 & 48.6 & 253 & 59.7 & 825 & 51.6 & & & \\
\hline \multirow{2}{*}{$\begin{array}{l}\text { Defending goal } \\
\text { post }\end{array}$} & $1^{\text {st }}$ goal post & 677 & 57.6 & 207 & 48.8 & 884 & 55.3 & 9.64 & 0.002 & $\varphi=-0.078$ \\
\hline & None & 499 & 42.4 & 217 & 51.2 & 716 & 44.8 & & & \\
\hline \multirow{2}{*}{$\begin{array}{l}\text { Type } \\
\text { marking }\end{array}$} & Zonal & 72 & 6.1 & 30 & 7.1 & 102 & 6.4 & 0.47 & 0.491 & $\varphi=-0.017$ \\
\hline & Combined & 1104 & 93.9 & 394 & 92.9 & 1498 & 93.6 & & & \\
\hline \multirow{2}{*}{$\begin{array}{l}\text { Intervening } \\
\text { attackers }\end{array}$} & $0-1$ & 608 & 51.7 & 3 & 0.7 & 611 & 38.2 & 343.30 & 0.000 & $\varphi=0.463$ \\
\hline & $>1$ & 568 & 48.3 & 421 & 99.3 & 989 & 61.8 & & & \\
\hline
\end{tabular}




\begin{tabular}{|c|c|c|c|c|c|c|c|c|c|c|}
\hline \multirow{2}{*}{$\begin{array}{l}\text { Defenders } \\
\text { inside box }\end{array}$} & $1-8$ & 391 & 33.2 & 125 & 29.5 & 516 & 32.3 & \multirow[t]{2}{*}{2.02} & \multirow[t]{2}{*}{0.155} & \multirow[t]{2}{*}{$C C=0.036$} \\
\hline & $>8$ & 785 & 66.8 & 299 & 70.5 & 1084 & 67.8 & & & \\
\hline \multirow{2}{*}{$\begin{array}{l}\text { Attackers } \\
\text { inside box }\end{array}$} & $1-5$ & 800 & 68.0 & 281 & 66.3 & 1081 & 67.6 & \multirow[t]{2}{*}{0.43} & \multirow[t]{2}{*}{0.508} & \multirow[t]{2}{*}{$\varphi=-0.017$} \\
\hline & $>5$ & 376 & 32.0 & 143 & 33.7 & 519 & 32.4 & & & \\
\hline \multirow{2}{*}{$\begin{array}{l}\text { Player of } \\
\text { contact }\end{array}$} & Defender & 978 & 83.2 & 102 & 24.1 & 1080 & 67.5 & \multirow[t]{2}{*}{496.29} & \multirow[t]{2}{*}{0.000} & \multirow[t]{2}{*}{$\varphi=0.557$} \\
\hline & Attacker & 198 & 16.8 & 322 & 75.9 & 520 & 32.5 & & & \\
\hline \multirow{4}{*}{ Outcome } & Attempt off target & - & - & 267 & 63.0 & 267 & 16.7 & \multirow[t]{4}{*}{-} & \multirow[t]{4}{*}{-} & \multirow[t]{4}{*}{-} \\
\hline & Attempt on target & - & - & 112 & 26.4 & 112 & 7.0 & & & \\
\hline & Goal & - & - & 45 & 10.6 & 45 & 2.8 & & & \\
\hline & No attempt & 1176 & 100 & - & - & 1176 & 73.5 & & & \\
\hline
\end{tabular}

* denotes significant difference between corner kicks with FAtt and NFAtt

Table 3. Logistic regression results based on teams' effectiveness.

\begin{tabular}{llllll}
\hline Variables & B & SE & Wald & Sig & Exp (B) [95\% CI] \\
\hline Direction of the ball & 3.19 & 2.07 & 2.37 & 0.12 & $24.32[0.42-1409.74]$ \\
Defending goal post & -0.19 & 0.14 & 1.84 & 0.17 & $0.82[0.62-1.09]$ \\
Type of marking & -0.35 & 0.30 & 1.34 & 0.24 & $0.69[0.38-1.28]$ \\
Intervening attackers & 4.14 & 0.59 & 48.36 & 0.00 & $63.05[19.61-202.74]$ \\
Defenders inside box & 0.19 & 0.20 & 0.91 & 0.34 & $1.21[0.81-1.80]$ \\
Attackers inside box & 0.27 & 0.17 & 2.59 & 0.10 & $1.31[0.94-1.84]$ \\
Player of the $1^{\text {st }}$ contact & 1.95 & 0.14 & 170.79 & 0.00 & $7.05[5.26-9.44]$ \\
Constant & -8.31 & 2.26 & 13.43 & 0.00 & 0.00 \\
\hline
\end{tabular}

\subsection{Binary logistic regression}

Binary logistic regression revealed that the model was statistically significant for FAtt $(667.443, p$ $<0.001$ ) and its predictive power was $R^{2}=49.8 \%$. The Hosmer-Leme show test, indicating goodness of fit, was not significant for the model $\left(x^{2}=4.439, p=\right.$ 0.815). The Wald statistic showed that the intervening attackers and the player of the 1st contact contributed significantly to the model (Table 3 ).

Specifically, the positive sign for the number of intervening attackers $(B=4.14, p=0.000)$ and the $O R$ of 63.05 , indicating that the likelihood of a team to execute a FAtt after corner kick increases with the number of intervening attackers. The positive sign for the player of $1^{\text {st }}$ contact $(B=1.953, p=0.000)$ and the OR of 7.05 indicates that the likelihood of FAtt increases 7-fold when an attacker accomplishes the first contact of the ball after corner kick.

The model in logit units is expressed in Equation 1:
Logit $=-8.313+4.144$ (Intervening attackers) +1.953 (Player of 1st contact)

Finally, the binary logistic regression revealed that the rest of the variables (Laterality, Direction, Marking type, Defending post, Interaction, defensive player and offensive player in the box) didn't contributed significantly to the model.

\section{Discussion}

The aim of the current study was to examine the characteristics of direct corner kicks and to define the variables related to corner's efficiency in Greek Super League 2018-19. The results of the present study suggest that corner kicks are a critical situation on creating final attempts, which could lead to goal scoring and eventually affect the final result of a match. More accurately, 1600 direct corner kicks were analyzed that were executed in 240 matches (6.7 corner kicks/match). Descriptive analysis demonstrated that 424 cases (26.5\%) led to final attempts and $2.8 \%$ 
resulted in goal. On the contrary, in $73.5 \%$ of the corners did not led to final attempt. These findings are consistent with similar results of previous studies, which investigated the long corner in the English Premier League [9] and confirmed the findings of de Baranda and Lopez-Riquelme [10] and Taylor, James and Mellalieu [12].

Nevertheless, even if this portion of corner kicks that resulted in goal seems small, this not contradicts the fact that corner kicks' efficiency plays an important role for the final outcome of the match. This is supported at a major extend from this study, as in $73.1 \%$ of the matches, the teams that executed efficiently direct corner kicks won or drawn the match. As far the situation of the match before corner kicks is concerned, results indicated that in most of the cases that corners led to final attempt, the current score was draw (53.5\%) and took place at the second half. This is in agreement with a previous study of Armatas and his colleagues [23]. From this, it could be inferred that teams in a winning status have the tendency to play more offensively and take more risks to win. Moreover, the increased portion of executed corners at the second half could be attributed to the respective increased physical and cognitive fatigue of the defensive players which forces them to commit a bigger number of errors during the game [3]. In other situational variables, Zhou and his colleagues [24] investigated Chinese Super League 2015 and found that better teams (higher rank) won more corner kicks, while home teams had positive small effect on corner kicks won.

With respect to the characteristics of the corner kick execution, results showed that outswing execution was preferred predominantly $(59.7 \%)$ and by significant difference $(p<0.001)$ from its counterparts, when after corner followed a final attempt, in comparison with cases without final attempt $(51.4 \%)$. This finding comes in contradiction with a previous study, which advocates that inswing corner executions present greater possibility for final attempt occurrence [12]. However, in the current study the outswing kick of the ball had as a consequence more final attempts out of target primarily delivered in the "front zone" (49.1\%) and the area "out of the box" $(17.5 \%)$, as it is shown in Figure 2. This could be possibly justified from the fact that the area near the goal and especially in the near post is occupied significantly by defenders, who intercept final attempts of the attackers. On the other hand, the area away from the goal provides more time and space for the attackers to execute $[3,9]$. This is confirmed by the presence of many defenders in the box $(>8)$ in $67 \%$ of the corner kicks, who were implementing a mixed defense in $93.6 \%$ of the cases, while in $57.6 \%$ of them at least one defender was positioned in the near post area, something that reduced significantly the chance for a final attempt. Considering the number of attacking players, one to five players were positioned in the box at $>65 \%$ of corner kicks, but without impact on the execution of the final attempt.

Most significant variables which affected the execution of the final attempt were "player of the 1st contact" and "intervening attackers". Descriptive analysis indicated that from the total number of direct corners, $75.9 \%$ ended with a final attempt, when the first player who received the ball in the box was an attacker, while in $99.3 \%$ more than two attackers were involved to claim the ball. This finding is also supported by the regression analysis, which indicated that the likelihood of a team to execute a FAtt after corner kick increases with the number of intervening attackers but also the likelihood of FAtt increases 7-fold when an attacker accomplishes the first contact, according to the predictive model below presented in Equation 1.

Finally, as it was mentioned before, from the total number of direct corner kicks, it was observed that approximately one fourth of them $(26.5 \%, 424$ corner kicks) ended with final attempt and in $2.8 \%$ of the cases a goal was scored. From them, 267 (63\%) were attempts off target, 112 (26.4\%) were attempts on target and $45(10.6 \%)$ resulted in goal. Similar findings were presented by Pulling [9] and de Baranda and Lopez-Riquelme [10]. On 2017/2018 FA Women's Super League, the $4.6 \%$ of corners taken resulted in a goal (in total 38 goals were scored), accounting for $13.5 \%$ of the total 282 goals scored during this season [25]. In a recent study from $\mathrm{Li}$ and Zhao [26], comparing KPS's between the "Big five" European football leagues, it was presented that more corner kick goals were scored in English Premier League (EPL) compared to the rest of the leagues. The authors claimed that the superb heading ability shown by EPL defenders may increase the number of corner kick goals.

\section{Conclusion}

To recapitulate, the main conclusions that could extracted from the current study are: 1 ) the efficiency of the corner kicks gets a small portion $(2.8 \%)$, although it could determine the match outcome, 2) the execution of final attempts as a 
consequence of corner kicks takes place in the central area between the goal area and the box, but in a significant percentage out of this $(17 \%), 3$ ) the likelihood of final attempt after corner kick increases when the number of intervening attackers increases and 4) the likelihood of final attempt after corner kick increases when an attacker accomplishes the first contact. Therefore, football coaches should devote more time in designing their training programs, offensively and defensively, for optimizing the efficiency of corner kicks executed by their team, as they could determine the final outcome in an elite football match.

\section{References}

[1] Hewitt, G. Greenham, K. Norton, Game style in soccer: what is it and can we quantify it?, International Journal of Performance Analysis in Sport, 16 (1) (2016) 355-372. [DOI]

[2] Kubayi, Analysis of Goal Scoring Patterns in the 2018 FIFA World Cup, Journal of Human Kinetics, 71 (1) (2020) 205-210. [DOI]

[3] C.A. Casal, R. Maneiro, T. Ardá, J.L. Losada, A. Rial, Analysis of corner kick success in elite football, International Journal of Performance Analysis in Sport, 15 (2) (2015) 430-451. [DOI]

[4] M. Mitrotasios, V. Armatas, Analysis of Goal Scoring Patterns in the 2012 European Football Championship, The Sport Journal, 1 (2014) 111.

[5] R. Maneiro, T. Ardá, A. Rial, J.L. Losada, C.A. Casal, S. López-García, Análisis descriptivo y comparativo de los saques de esquina. UEFA Euro 2012, Revista Andaluza de Medicina del Deporte, 10 (3) (2017) 95-99. [DOI]

[6] A. Vergonis, Y. Michailidis, T. Metaxas, The Significant Role of Scoring from Set Plays in the 2018 FIFA World Cup, International Scientific Journal of Kinesiology, 47 (2021) 4751. [DOI]

[7] J. Lago, C. Lago, E. Rey, Relevancia de las acciones a balon parado sobre los resultados en el Mundial 2006, Training Futbol, 134 (2007) 46.

[8] A.M. Luongo, (1996) The soccer handbook for players, coaches and parents. McFarland, London, U.K.

[9] Pulling, Long corner kicks in the English premier league: deliveries into the goal area and critical area, Kinesiology 47 (2) (2015) 193-201.

[10] S.P. de Baranda, D. Lopez-Riquelme, Analysis of corner kicks in relation to match status in the 2006 World Cup, European Journal of Sport Science, 12 (2012) 121-129. [DOI]

[11] Carling, A.M. Williams, T. Reilly (2005) Handbook of soccer match analysis: A systematic approach to improving performance, Routledge, Abingdon, UK.

[12] J. Taylor, N. James, S.D. Mellalieu, Notational analysis of corner kicks in English Premier League soccer, Journal of Sports Sciences, 22 (6) (2004) 518-519.

[13] R. Castillo, F.A. Cruz, A. Raya, J.M. Castillo, Análisis técnico-táctico en los corners del Mundial de Francia-98, Training Fútbol, 49 (2000) 14-23.

[14] A. Grant, M. Williams, Analysis of corner kicks, Insight, 1 (3) (1998) 25-26.

[15] S. Poon, A. Douglas, W.G. Hopkins, Notational analysis of long corner kicks in an international youth football tournament, International Journal of Performance Analysis in Sport, 12 (3) (2012) 692.

[16] Pulling, M. Robins, T. Rixon, Defending corner kicks: analysis from the English Premier League, International Journal of Performance Analysis in Sport, 13 (1) (2013) 135-148. [DOI]

[17] V.A. Miracle, The Belmont Report: The triple crown of research ethics, Dimensions of Critical Care Nursing, 35 (4) (2016) 223-228. [DOI] [PubMed]

[18] Gabin, O. Camerino, M.T. Anguera, M. Castañer, Lince: Multiplatform Sport Analysis Software, Procedia-Social and Behavioral Sciences, 46 (2012) 4692-4694. [DOI]

[19] Pulling, J. Newton, Defending corner kicks in the English Premier League: near-post guard systems, International Journal of Performance Analysis in Sport, 17 (3) (2017) 283-292. [DOI]

[20] J.L. Losada, R. Manolov, The process of basic training, applied training, maintaining the performance of an observer, Quality \& Quantity, 49 (1) (2015) 339-347. [DOI]

[21] G. Robinson, P. O'Donoghue, A weighted kappa statistic for reliability testing in performance analysis of sport, International Journal of Performance Analysis in Sport, 7 (1) (2007) 12-19. [DOI] 
[22] J. Cohen, (1988) Statistical power analysis for the behavioral sciences (2nd ed.), L. Erlbaum Associates, Hillsdale, N.J., USA.

[23] V. Armatas, Y. Yiannakos, S. Papadopoulou, C. Galazoulas, Analysis of the set-plays in the $18^{\text {th }}$ football World Cup in Germany, Physical Training, 1 (2007) 10-17.

[24] Zhou, W.G. Hopkins, W. Mao, A.L. Calvo, H. Liu, Match performance of soccer teams in the Chinese Super League-effects of situational and environmental factors, International Journal of Environmental Research and Public Health, 16 (21) (2019) 4238. [DOI] [PubMed]

[25] H. Beare, J.A. Stone, Analysis of attacking corner kick strategies in the FA women's Super League 2017/2018, International Journal of Performance Analysis in Sport, 19 (6) (2019) 893-903. [DOI]

[26] C. Li, Y. Zhao, Comparison of goal scoring patterns in "The Big Five" European Football Leagues, Frontiers in Psychology, 11 (2021) 619304. [DOI] [PubMed]

\section{Funding}

No funding was received for conducting this study.

\section{Conflict of interest}

The Author has no conflicts of interest to declare that they are relevant to the content of this article.

\section{Does this article screened for similarity?}

Yes

\section{About The License}

(C) The Author 2021. The text of this article is open access and licensed under a Creative Commons Attribution 4.0 International License 